INT. J. PROD. RES., 2000, VOL. 38, NO. 15, 3603-3621

\title{
Defining specialized design similarity measures
}

\author{
JEFFREY W. HER R MANN $\dagger^{*}$, SUNDAR BALASUBR AMANIAN $\dagger$ \\ and GURDIP SINGH†
}

\begin{abstract}
Similar product designs resemble each other and have similar attributes and characteristics. When examining a product design to create its process plan, design its fixture, or estimate its manufacturing cost, manufacturing engineers often identify one or more similar products that the factory has manufactured in the past. They may do this from memory, from a file cabinet, or from a database (in a product data management system). Then, they retrieve the process plan (or fixture or cost estimate) for the similar product and modify it for the new product. Manufacturing and process planning experts use a complex set of rules, guidelines, and other knowledge to determine how similar two products are. Computer-aided process planning tools, however, generally use simpler, less sophisticated procedures for determining similarity. These traditional procedures may be inappropriate for specific settings. This paper presents an approach for developing function-specific design similarity measures. Such a measure explicitly exploits the specific need for similar products and thus improves variant approaches for process planning, fixture planning, and manufacturability evaluation. The approach is applied to a specific fixture planning domain.
\end{abstract}

\section{Introduction}

Reusing existing solutions is an efficient approach to many manufacturing tasks, including process planning, fixture planning, and manufacturability evaluation. Given a new product design and a task to perform, many manufacturing engineers start by identifying one or more similar products. They may search their memory, file cabinets, or databases (in a product data management system). After locating a similar product, they can use information about that product to create information about the new product or predict the new product's performance. For instance, variant process planning modifies a similar product's process plan to create the new product's process plan. Variant fixture planning modifies a similar product's fixture to create the new product's fixture. Variant manufacturability evaluation uses a similar product's manufacturing cost or time to predict the new product's manufacturing cost and time.

Engineers have, in the past, been successful by trusting their experience and intelligence when locating similar products. The variety of products and manufacturing processes continues to grow, however. Thus, manufacturing companies need systematic procedures so that fewer mistakes occur, decisions conform to standard practice, and new engineers can perform the job efficiently and effectively. In addi-

Revision received March 2000.

$\dagger$ Department of Mechanical Engineering and Institute for Systems Research, University of Maryland, College Park, MD 20742, USA.

* To whom correspondence should be addressed. e-mail: jwh2@eng.umd.edu 
tion, systematic procedures can be automated, reducing the time that engineers spend doing routine tasks.

A systematic method for retrieving a similar product requires some way to classify or organize the set of existing products. We will use the term design similarity measure to describe a function or procedure that calculates or estimates the similarity of two product designs. Researchers and software developers have constructed some approaches for classifying product designs according to their similarity, and these have been used in some particular domains as the basis for computer-aided variant process planning. Section 2.2 will discuss these in more detail.

Computer-aided process planning tools, however, generally use simple procedures for determining similarity. These traditional procedures may be inappropriate for specific settings. Moreover, no previous work has presented a general approach for defining a specialized design similarity measure. This deficiency hampers engineers and researchers who attempt to develop variant procedures for other problem domains. If one knew how to define the appropriate design similarity measure, one could deploy a systematic procedure for locating a similar product to help complete a manufacturing task (like process planning, fixture planning, or manufacturability evaluation) more efficiently.

Consider the following analogy. A commuter is shopping for a new car. The commuter is considering a particular new car and wants to estimate the car's actual fuel efficiency. The commuter knows a lot about the new car and knows a lot about other existing cars and their actual fuel efficiency in the commuter's community. The commuter believes that the new car's fuel efficiency will approach the fuel efficiency of similar cars. Specifically, the car whose weight most closely approaches the new car's weight will provide the best estimate for the new car's fuel efficiency. The commuter ignores the car's colour and the stereo (which do not impact the fuel efficiency) and instead considers the car's weight (which does).

The commuter's car similarity measure is a function of the two car's weights. As the weights approach each other, the cars' similarity increases. The commuter uses this measure to identify the most similar car and estimate the new car's fuel efficiency. Of course, the commuter might find a better estimate by also considering the car's engine displacement and whether or not it has air conditioning, since those attributes also affect fuel efficiency. Also, note that the similarity measure does not itself estimate the fuel efficiency; instead, it helps identify cars that may have similar fuel efficiency.

The manufacturing engineer's problem can be formulated as follows: The decision-maker has a set $W$ of product designs and relevant information about those product designs. Let $\left(D_{i}, L_{i}\right)$ be a product design and the relevant information (for example, the process plan). The decision-maker has a new product design $D_{0}$ and needs to create some information $L_{0}$ about that product design. The decision-maker wants to identify a product design in $W$ and use the associated information to create $L_{0}$. One can say that $L_{0}$ solves the decision-maker's problem.

To do this, the decision-maker needs a design similarity measure $S\left(D_{0}, D_{j}\right)$ that quantifies how well the information $L_{j}$ can be used to create some information $L_{0}$ about $D_{0}$. The decision-maker can then search $W$ for the existing product design in $W$ that is most similar to $D_{0}$. We will assume that $S\left(D_{0}, D_{j}\right)$ ranges from zero to one. If $S\left(D_{0}, D_{j}\right)$ is close to 0 , then $L_{j}$ is not very useful. If $S\left(D_{0}, D_{j}\right)$ is close to 1 , then $L_{j}$ is very useful. (For example, perhaps the process plan $L_{j}$ for product $D_{j}$ requires only minor modifications to be a feasible process plan for $D_{0}$.) 
The proper design similarity measure is important since it can help the decisionmaker construct a superior solution quickly. An improper design similarity measure will lead the decision-maker to an inferior solution or will cause delays as the decision-maker searches $W$ exhaustively to find a better source of information.

An alternative approach would determine directly how well the information $L_{j}$ can be used for $D_{0}$. In some cases, this may be impossible or computationally expensive. For example, if $L_{j}$ is the manufacturing cost of $D_{j}$, then it is difficult to determine independently whether $L_{j}$ is an accurate estimate for the manufacturing cost of $D_{0}$. If $L_{j}$ is a fixture for $D_{j}$, then it will require significant computational effort to determine directly how well $L_{j}$ would hold $D_{0}$. However, a design similarity measure, in general, will be feasible and will require less computation.

If the decision-maker needs to create this type of information frequently, then the decision-maker may find it worthwhile to invest some time creating a specialized design similarity measure that helps solve this type of problem. For instance, a manufacturing engineer may decide to create a design similarity measure that identifies product designs that will have similar manufacturing costs or times. This specialized design similarity measure will be different from a measure used to locate a product design that has a similar fixture or process plan.

There are many ways to define a specialized design similarity measure. One can try to capture the expert knowledge that an experienced engineer has and to store the rules and information in a knowledge base to develop an expert system that can calculate $S\left(D_{0}, D_{j}\right)$. Herrmann and Singh (1997) describe an approach that structures the expert's knowledge and constructs a plan-based design similarity measure for variant process planning.

A second approach is to exploit the knowledge that exists in the set $W$. This paper discusses such an approach to define a specialized design similarity measure. This approach uses an artificial neural network. The product designs and information in $W$ are used to train the neural network, which learns the specialized design similarity measure. We present an application of this approach to the domain of variant fixture planning. However, the approach could be used for variant process planning or variant manufacturability evaluation.

The remainder of this paper describes the approach in detail. Section 2 reviews related work on process planning, design similarity measures, fixture planning, and neural networks. Section 3 describes the approach for defining a specialized design similarity approach. Section 4 presents the application of this approach to variant fixture planning. Section 5 concludes the paper.

\section{Related work}

This section reviews previous work on process planning, design similarity measures, fixture planning, and artificial neural networks.

\subsection{Process planning}

A process plan describes the steps necessary to manufacture a product. It specifies the type and sequence of the manufacturing operations that transform the components and raw materials into a set of parts. Some form of the process plan is used by designers, production planners, and manufacturing operators. A process plan may specify, for each step, the particular machine that will perform the step and the relevant process parameters that regulate the process. For instance, the process plan may specify the speed required to perform a drilling operation. 
The earliest examples of process plans are operations sheets for sewing machine manufacture. In the nineteenth century, Brown \& Sharpe, a Rhode Island manufacturing company, used these sheets to list the operations and identify, for each operation, the necessary tools, jigs, fixtures and gauges (Hounshell, 1984).

When done manually, process planning is a subjective and time-consuming procedure, and it requires extensive manufacturing knowledge. An experienced engineer or machinist must examine a part drawing and construct a process plan. The process planner must know or be able to find information about the manufacturing capabilities, tooling, materials, costs, and machine availability. In addition, the process planner must carefully document the plan using standard notation and forms.

Computer-aided process planning (CAPP) software systems automate many functions, which reduces the chance of error, and the process planner can work more quickly. Variant process planning systems have successfully exploited the observation that similar designs have similar process plans. Thus, the most similar design will have the most useful plan. (A useful plan is one that requires few changes.) The CAPP software searches a set of existing designs, uses a design similarity measure to compare each existing design and the new design, identifies the most similar existing design, and retrieves the corresponding process plan. (Some variant process planners find the most similar product family and retrieve that family's standard plan.) Then, the process planner modifies the process plan, changing the details so that the plan is appropriate for the new design. The new plan is therefore a variant of the old plan.

The earliest CAPP software systems employed the variant process planning technique. Several variant systems are commercially available today, and they currently support almost all practical implementations of CAPP. For more information, see, for example, Chang and Wysk (1985), Alting and Zhang (1989), Bedworth et al. (1991), and Halevi and Weill (1995). A typical variant process planning approach involves the following three steps.

(1) The process engineer uses a group technology (GT) coding scheme to map a new product design $D_{0}$ into an alphanumeric code. As discussed below, GT codes are a popular design classification scheme.

(2) This code is then used as an index into a database to retrieve a process plan $P$ for the design $D$ most similar to $D_{0}$ (or for the family $F$ that includes $D_{0}$ or is most similar to $D_{0}$ ).

(3) The planner then modifies the process plan $P$ manually to produce a plan $P_{0}$ for the design $D_{0}$.

Variant process planning is a popular technique and several available CAPP systems have implemented the approach. It has many advantages.

(i) Because the planner only modifies the retrieved plan, variant process planning reduces the total time that the planner requires to generate a complete process plan for the new design.

(ii) The process plan uses existing company manufacturing data and expertise and follows company standards.

(iii) The variant approach can be applied to any type of manufacturing, and the resulting plan can include all types of processes. 
Truly effective variant process planning requires the ability to retrieve a process plan that is useful for creating the new plan. Since the retrieved process plan corresponds to the most similar design (or family) that the search finds, the design similarity measure clearly affects the variant process planning approach. However, existing design similarity measures do not consider explicitly the process plan attributes. This may lead to instances when similar' designs have process plans that are less useful.

Most variant approaches use product (or part) families to simplify the procedure. A new product design must be classified into the most appropriate product family. The next section discusses different approaches for forming the families and classifying a product design into a family.

\subsection{Design similarity measures}

This section reviews the common design similarity measures that group technology and variant process planning use. Other approaches include geometric similarity and neural network classifiers.

Group Technology. Mitrofanov (1966) first formally described group technology (GT) as a method that improves manufacturing efficiency by classifying similar products into families. Two formal methods of classifying designs for group technology are Production Flow Analysis (PFA), described by Burbidge (1989), and parts coding and classification analysis (PCA). PFA uses routing information to classify products into families, while PCA uses design information to derive GT codes.

A GT code is a sequence of numbers. Each position in the code represents some product design attribute: thickness or the presence of holes, for example. Each possible value represents a set of values for that attribute. Different GT coding schemes have different attributes. Given a product design and a specific coding scheme, the coding rules calculate what each attribute's value should be, which yields a GT code for that product. Researchers have developed many GT coding schemes. Typical schemes include DCLASS (Computer Aided Manufacturing Laboratory, 1979), MICLASS (Houtzeel and Schilperoort, 1976), and OPITZ (Opitz, 1970). Also see, for example, Chang and Wysk (1985) and Bedworth et al. (1991). GT codes have many applications, including cellular manufacturing system design, materials management, tool management, process planning, and product standardization (Snead, 1989). Candadai et al. (1995, 1996) describe an application for manufacturability evaluation and partner selection.

The PFA approach examines the product routings and then groups those products that have similar routings. Usually, one can use clustering algorithms such as the rank order cluster algorithm (King, 1980) or mathematical formulations (Kusiak, 1987) to identify similar routings and classify products into families.

Variant process planning requires some way to represent the products that each family contains. If each product has a code, the part family matrix (Chang and Wysk, 1985) describes, for each GT code position, all possible values that members of that family have. Thus, a new product belongs to a particular family if the GT code for the new design has, in each position, a value that exists in that family's part family matrix. That family's standard process plan becomes the new product's process plan. 
PFA uses a rational approach to form families whose products have similar routings. The subsequent search uses a GT code to classify a new product and then asserts that the GT code similarity implies that the new product should have a similar process plan. However, the GT code may not correspond to the process plan. Although the GT code captures some information that impacts manufacturing, the code does not explicitly describe the process plan. Thus, GT codes may be convenient for classification, but their use in this approach is inconsistent.

The PCA approach uses the GT codes to classify products into families. Offodile (1991) describes one such approach. The approach uses a similarity measure to evaluate each pair of products and calculate their similarity. This measure uses each product's GT code and averages the code digits' similarity. A single linkage clustering algorithm then groups the products into families at different similarity thresholds. Each family has a part family matrix and a standard process plan. As before, a new product belongs to a particular family if the GT code for the new product has, in each position, a value that exists in that family's part family matrix. That family's standard process plan becomes the new product's process plan. Although Offodile's approach simply averages the absolute digit differences, Iyer and Nagi $(1994,1997)$ describe a more flexible GT code-based search.

The PCA design classification approach is consistent because the search uses the same criteria that the part family grouping does. Again, however, a product's GT code may not correspond to its process plan. It is not clear that the family's standard process plan would be a useful process plan for the new product. Thus, this may be an inappropriate variant process planning approach.

Geometric Approaches. Many modern CAD/CAM systems use constructive or boundary models to represent solids. Thus, one can classify products by using the geometry that the CAD models represent. Elinson et al. (1997) describe such approaches in detail.

Many solid models use Constructive Solid Geometry (CSG) trees as a basic representational scheme. Using CSG trees to classify products seems natural because the component volumes may be the volumes that machining operations remove. However, the approach has two drawbacks. First, a product may have more than one CSG representation. Second, although similar, the CSG primitives do not always correspond to the manufacturing features. When they do not, the CSG tree does not describe the information relevant to process planning.

Sun et al. (1995) have described a similarity measure for solids. This measure evaluates properties of their boundary representations. This is an interesting, new measure of relaxed' geometric similarity. However, it has several difficulties that limit its use as a classification scheme for manufacturing. First, the similarity measure can compare only polyhedral objects. Thus, the approach must facet (approximate with planes) a solid that has cylindrical or sculpted faces. It is unclear how this approximation will affect the design similarity measure. The second difficulty is that the current measure addresses no manufacturing considerations such as approachability, operation interference, or fixturing. It is unclear if the design similarity measure can include these considerations.

Neural networks. One promising approach (Leung et al. 1994) exploits the strengths of the Artificial Neural Network (ANN) technique to associate a product's attributes with its process plan attributes. This approach does not use an explicit design similarity measure but implicitly maps the product attributes to the associated process 
plan attributes. Thus, this approach is more like a generative method. However, the resulting process planning systems have yielded inconsistent results since the search's success depends upon the ANN technique and the sample data used to train the network.

Other design classification approaches use neural networks to solve the form recognition problem (Wu and Jen 1996). However, such approaches resemble the geometric approaches and share the same drawbacks.

Expert system. Herrmann and Singh (1997) describe a three-step approach for defining a plan-based design similarity measure using expert knowledge about the process planning domain. First, the expert defines a process plan similarity measure that is a function of selected process plan attributes. For instance, the relevant attributes might be the presence of specific manufacturing operations, and the similarity measure might describe how many manufacturing processes the two process plans share. In the second step, the expert selects product design attributes. Then, for each process plan attribute in the process plan similarity measure, the expert defines a mapping function that describes the correlation between the product design attributes and the process plan attribute. The third step constructs the design similarity measure by substituting the mapping functions into the process plan similarity measure.

\subsection{Fixture planning}

This section reviews some of the previous research into fixture planning.

In machining and other types of operations, an important part of process planning is fixture planning: determining the fixture that holds, locates, and supports a workpiece in a particular set-up and provides a means to reference and align the cutting tool to the workpiece. The proper location of the workpiece is essential to guarantee accuracy and repeatability of the machining process (Hoffman 1984).

Fixture planning is an important issue in small-batch manufacturing, which requires the flexibility of modular fixtures. While many areas have been explored to improve the cost-effectiveness of a manufacturing activity, none have an impact on productivity as significant as workholding practice (Hoffman 1987). Like process planning, identifying a good fixture for a given operation is a difficult task. Fixture planning is difficult because there are many different types of fixtures and fixture elements, and the fixture has to satisfy many constraints on stability, location, restraint, accessibility, and cost.

Process planning and fixture planning are two problems that have been addressed separately, but they have a significant effect on each other. Many researchers have attacked the problem of automatic fixture design and the integration of fixture planning and process planning. Previous research has focused on mathematical solutions for locating and holding a part and on expert systems and computer-aided fixture-planning routines. See Darvishi and Gill (1990), Trappey and Liu (1990), Chang (1992), Kumar et al. (1992), Fuh et al. (1993), Hargrove and Kusiak (1994), Yue and Murray (1994), and Chou et al. (1994) for some approaches and additional references on automatic fixture design.

\subsection{Artificial neural networks}

Artificial neural networks are computer simulations of biological neurons, composed of nonlinear computational elements operating in parallel. Neural net- 
works consist of nodes (neurons) and synaptic connections that connect these nodes. Each connection is assigned a relative weight, also called connection strength, or synaptic strength. The output at each node depends on the threshold (bias or off set) specified and a transfer (activation) function. For a complete introduction, see Muller and Reinhardt (1990). See Lippmann (1987) for a taxonomy of neural network structures.

Some typical applications of neural networks involve pattern mapping, pattern completion and pattern classification. Neural networks attract interest because these models are capable of performing complex tasks that are impossible with sequential models. Neural networks are particularly useful in problems where the logical structure or the inputoutput relation is poorly understood. Neural networks are capable of learning and generalizing from examples in the absence of explicit rules or an analytical structure.

In a feed-forward neural network, signals flow from the input layer through the intermediate hidden layers to the output layer. Neurons in the same layer do not communicate with each other. Multi-layered networks have input, output, and inner (or hidden) neuron layers that intervene between the input and output layers. The number of hidden layers and number of nodes in each hidden layer depends on the complexity of the problem. Increasing the number of hidden layers increases the network's complexity and may or may not enhance the network's performance. In most cases, a hidden layer that has more nodes yields better network performance and longer training time. Based on previous experience, one or two hidden layers provide a better performance while not requiring extensive training time (Huang et al. 1999).

The initial neural network has a random set of weights. The learning process adjusts the connection weights to improve the neural network's performance on a predefined measure. Supervised learning presents a set of training input vectors and their associated output vectors. The learning algorithm adjusts the network's weights based on the difference between the desired output and neural network's actual output. Learning is a search, through a multi-dimensional space of weights, that gradually optimizes the network performance (Hassoun 1995). Back-propagation is the most extensively used training method. The back-propagation algorithm is an iterative gradient method-based algorithm developed to introduce synaptic corrections (weight adjustments) by minimizing the sum of squared error (objective function).

\section{Approach}

This section describes an approach for creating a specialized design similarity measure. This approach uses an artificial neural network. The existing designs and information are used to train the neural network, which learns the specialized design similarity measure. We will present an application of this approach to the domain of variant fixture planning. However, the approach is also appropriate for variant process planning and variant manufacturability evaluation. Defining a specialized design similarity measure requires some effort. Once done, it yields a more appropriate measure that is quick and easy to use. The key is to use the knowledge that exists in $\mathrm{W}$, the set of existing product designs and information. The approach proceeds as follows. 
Step 1. Define a function $F\left(D_{i}, L_{i}, D_{j}, L_{j}\right)$ that quantifies whether the information $L_{j}$ is appropriate for product design $D_{i}$. The function should range from 0 to 1 , with $F=0$ signalling that $L_{j}$ is completely inappropriate for $D_{i}$, and $F=1$ signalling that $L_{j}$ is perfect for $D_{i} . F\left(D_{i}, L_{i}, D_{i}, L_{i}\right)$ should equal 1 . Note that $F\left(D_{i}, L_{i}, D_{j}, L_{j}\right)$ does not necessarily equal $F\left(D_{j}, L_{j}, D_{i}, L_{i}\right)$.

For example, if $L_{j}$ is the process plan for $D_{j}, F$ might represent the fraction of operations in $L_{j}$ that are needed for $D_{i}$ and in $L_{i}$.

Step 2. For all pairs $i, j$ in $W \times W$, calculate $U_{i j}=F\left(D_{i}, L_{i}, D_{j}, L_{j}\right)$. (Note $U_{i i}=1$.) Create a set $X=\left\{\left(D_{i}, D_{j}, U_{i j}\right)\right.$ : for all $i, j$ in $\left.W \times W\right\}$.

Step 3. Construct a neural network that has one output node. Its input nodes correspond to the key design attributes of two designs. Initialize the weights with randomly selected values.

Step 4. Partition $X$ into two sets: a training set and a testing set. Use these sets to train the neural network. The input signals to the neural network are the attributes of the designs $D_{i}$ and $D_{j}$, and the desired output is $U_{i j}$. The resulting neural network is the specialized design similarity measure $S\left(D_{i}, D_{j}\right)$.

\section{Application to variant fixture planning}

One application for a specialized design similarity measure is our work on hybrid process planning.

\subsection{Hybrid process planning}

Variant fixture planning is part of a hybrid process planning approach that we have developed. The hybrid process planning approach extends the generative approach that Gupta et al. (1994) describe. After using that approach for process selection, it employs a variant procedure to select fixtures, which completes the process plan. For more details, see Balasubramanian et al. (1998).

In a machining operation, a cutting tool sweeps along a trajectory, and the motion of the tool relative to the current workpiece removes material. A machining feature is the volume resulting from a machining operation. A machining feature corresponds to a single machining operation made on one machine set-up. Each machining feature has a single approach direction (or orientation) for the tool.

Features are parametrized solids that correspond to various types of machining operations on a three-axis machining centre: side-milling, face-milling, end-milling and drilling. A design is represented as a collection of machining features. Given this feature-based representation, there may be several alternative representations of the design as different collections of machinable features, corresponding to different ways to machine the part. The approach proceeds as follows.

Repeat the following steps until every promising feature-based model (FBM) has been examined.

- Generate a promising FBM from the feature set. A FBM is a set of machining features that contains no redundant features and is sufficient to create the part. An FBM is unpromising if it is not expected to result in any operation plans better than the ones that have already been examined.

- Do the following steps repeatedly, until every promising operation plan resulting from the particular FBM has been examined. 
- Generate a promising operation plan for the FBM. This operation plan represents a partially ordered set of machining operations. We consider an operation plan to be unpromising if it violates any common machining practices.

- Estimate the achievable machining accuracy of the operation plan. If the operation plan cannot produce the required design tolerances and surface finishes, then discard it. Otherwise, estimate the production time and cost associated with the operation plan.

- For each set-up in the operation plan, design a fixture in the following way. Search a database of existing designs, process plans, and fixtures, for fixtures that could be used for the new design. Modify the retrieved fixture as necessary and verify its feasibility.

- If no promising operation plans were found, then exit with failure. Otherwise exit with success, returning the operation plan that represents the best trade-off among quality, cost and time.

\subsection{Variant fixture planning}

This section describes the variant fixture planning step in the hybrid process planning approach described above. The goal is to retrieve, for a new product design, a useful fixture from a given set (or database) of existing designs and their fixtures. Thus, the variant approach exploits this existing knowledge. However, since calculating each fixture's feasibility and then determining the necessary modifications for infeasible fixtures would require too much effort, the approach searches quickly for the most promising fixtures. As explained below, the proposed approach uses a design similarity measure to find existing designs that are likely to have useful fixtures. Then, it modifies the retrieved fixtures as necessary and identifies the best one for the new design.

In order to demonstrate our approach, we have studied a particular class of products and modular components. One face of the part rests on the supporting plane (a baseplate) and the fixture elements constrain all motion of the part in the supporting plane. Thus, only the two-dimensional projection of any given design onto the supporting plane is needed for fixture planning. Only polygonal shapes are considered. In this setting, a fixture is a set of three locators (pins) and one clamp, which provides form closure. Because generative fixture planning approaches (Brost and Goldberg 1996, Zhuang and Goldberg 1996) are available, this domain is a convenient environment for testing our approach. Future work will extend the variant fixture planning approach to a broader class of products and fixtures.

For a new product design, a fixture's usefulness is defined as its ability to provide form closure and its maximum contact reaction force under a unit torque. The reciprocal of this reaction force is the torque resistance metric. If the maximum contact reaction force is smaller, the metric is larger. Large contact reaction forces are undesirable since they may deform the part. Moreover, in the presence of large machining forces, large contact reaction forces can make large clamping forces necessary. Unfortunately, because calculating this measure requires some effort, checking each existing fixture against a new design is impractical if the database is large.

The following steps describe the approach concisely. More details follow. Let $D_{0}$ denote the new design. $W$ is the set of existing designs and fixtures. 
Step $0 . \Gamma=\{\} . \Gamma$ will be the set of candidate fixtures.

Step 1. For each $D_{j}$ in $W$, calculate $S\left(D_{0}, D_{j}\right)$. For the product designs $D_{j}$ that maximize $S\left(D_{0}, D_{j}\right)$, add $L_{j}$ (the fixture for $\left.D_{j}\right)$ to $\Gamma$.

Step 2. For each $L_{j}$ in $\Gamma$ :

Step 2a. Determine all feasible configurations of $D_{0}$ in the locator triplet of $L_{j}$.

Step $2 \mathrm{~b}$. For each feasible configuration, find the clamp positions that achieve form closure.

Step 2c. For each feasible clamp position (and configuration) $C$, evaluate the torque resistance metric $r\left(C, D_{0}\right)$.

Step 2d. Let $t\left(D_{0}, L_{j}\right)=\max r\left(C, D_{0}\right)$.

Step 3. Select the fixture $L_{j}$ in $\Gamma$ that maximizes $t\left(D_{0}, L_{j}\right)$. The design similarity measure $S\left(D_{0}, D_{j}\right)$ reflects fixture usefulness and was developed using the neural network approach described in Section 3.

\subsection{Defining the design similarity measure}

This section presents the specialized design similarity measure constructed for this variant fixture planning application. To do this, we followed the steps described in Section 3.

Set $W$ contains 27 two-dimensional product designs and the fixture for each. We used available generative fixture planning techniques to create each product's fixture. The existing fixtures provide the least maximum contact reaction force for an applied unit torque (clockwise or counter-clockwise). However, other metrics, such as force resistance or force and torque resistance, could be considered.

Step 1 . We defined the function $F\left(D_{i}, L_{i}, D_{j}, L_{j}\right)$ as the ratio of the torque resistance metric when fixture $L_{j}$ is used for product design $D_{i}$ to the torque resistance metric when fixture $L_{i}$ is used for product design $D_{i}$. Since $L_{i}$ is the best fixture for $D_{i}$ and has the maximum torque resistance metric, this ratio is in the range $[0,1]$.

Step 2. We calculated $U_{i j}=F\left(D_{i}, L_{i}, D_{j}, L_{j}\right)$ for all $729\left(27^{2}\right)$ pairs of product designs and created set $X$.

Step 3. We constructed a neural network with seven input nodes, one hidden layer with seven nodes, and one output node. Three input nodes correspond to the product design attributes of $D_{i}$, three input nodes correspond to the product design attributes of $D_{j}$, and the seventh is a bias node. Below we describe the neural network in more detail.

Step 4 . We partitioned $X$ by randomly selecting 546 pairs for the training set. The remaining 183 pairs formed the testing set. Using the back propagation algorithm, we trained the neural network. More details are given below. The resulting neural network formed our specialized design similarity measure $S\left(D_{i}, D_{j}\right)$.

The neural network structure. We used a feed-forward network with sigmoid activation functions for hidden and output layers. Learning is by the Least Mean Square-based Back Propagation Algorithm.

In this fixture planning domain, the product design attributes of interest were the Maximum Inter-vertex Distance (MID), the Maximum Vertex-Edge Distance (MVED), and the Total Enclosed Area (TEA). All of these are functions of the two-dimensional projection of the product design. The MID is the maximum dis- 
tance between any pair of vertices in the polygon. The TEA is the area enclosed by the polygon. The MVED is the maximum distance between a vertex and a line containing an edge of the polygon.

In many cases, pre-processing the variables is one of the most significant factors in determining the performance of the neural network model (Bishop 1995). It is often necessary to transform the input data into some new representation before presenting them to the network. In this application, we standardized the input variables by calculating, for the product designs in $W$, the mean and standard deviation of each product design attribute: MID, MVED and TEA. For training and testing purposes, we standardized each value by subtracting the appropriate mean and dividing by the standard deviation. Thus, in the training and testing sets, each attribute's mean equals zero and its standard deviation equals one.

Training and testing. A training cycle proceeds as follows. Present the first training example to the neural network and evaluate the actual output. Use the back-propagation algorithm to adjust the weights. Repeat for the remaining examples in the training set. Then, present the first testing example to the neural network and evaluate the actual output. Record the absolute value of the difference between the actual output and the desired output. Repeat for the remaining examples in the testing set and then average. This yields the Mean Absolute Deviation (MAD).

To train the neural network, we began by randomly initializing the weights in the neural network. We then performed training cycles until the MAD stopped improving.

As mentioned earlier, there is no methodology to select the number of hidden layers, number of nodes in each hidden layer, connectivity, or learning algorithm. Thus, we constructed different networks to find a superior configuration. We varied the number of hidden layers (one or two), the number of nodes in each hidden layer (seven or thirteen), and the learning rate, which affects the weight adjustment. We performed Steps 3 and 4 for each neural network design. The best network design had one hidden layer, seven nodes, and a learning rate of 0.09 . In all designs, we used a momentum factor of 0.9 . The resulting MAD was 0.206576 .

\subsection{Results}

We compared the specialized design similarity measure $S\left(D_{i}, D_{j}\right)$ to design similarity measures $P\left(D_{i}, D_{j}\right), Q\left(D_{i}, D_{j}\right)$ and $R\left(D_{i}, D_{j}\right)$, which were based on the individual attributes MID, TEA and MVED (as defined in Appendix A).

For every pair of designs $\left(D_{i}, D_{j}\right)$ in $W \times W$, we calculated $U_{i j}=F\left(D_{i}, L_{i}, D_{j}, L_{j}\right)$, the relative usefulness of fixture $L_{j}$ for design $D_{i}$. We also calculated the specialized design similarity measure $S\left(D_{i}, D_{j}\right)$ for every pair of designs. Using standard statistical techniques we computed the correlation coefficient for the pairs $\left(U_{i j}, S\left(D_{i}, D_{j}\right)\right)$. Similarly, we computed the correlation coefficient for the pairs $\left(U_{i j}, P\left(D_{i}, D j\right)\right)$, the correlation coefficient for the pairs $\left(U_{i j}, Q\left(D_{i}, D_{j}\right)\right)$, and the correlation coefficient for the pairs $\left(U_{i j}, R\left(D_{i}, D_{j}\right)\right)$. Table 1 presents these results. The largest correlation coefficient corresponds to the specialized design similarity measure $S\left(D_{i}, D_{j}\right)$, implying that this measure predicts fixture usefulness better than the simple design similarity measures.

We then examined how well the different similarity measures performed when used to select existing fixtures for new product designs. We created ten new product designs not in the set of existing product designs. For each one of these, we used the 


\section{Design}

similarity

measure

$P\left(D_{i}, D_{j}\right)$

$Q\left(D_{i}, D_{j}\right)$

$R\left(D_{i}, D_{j}\right)$

(MVED)

$S\left(D_{i}, D_{j}\right)$

Correlation

coefficient

0.3593

0.3432

0.1558

0.6001

Table 1. Correlation coefficients for design similarity and relative fixture usefulness.

specialized design similarity measure $S\left(D_{i}, D_{j}\right)$ to find the existing product design most similar to the new one and measured the usefulness of that existing product's fixture for the new product design. Table 2 displays the results. Similarly, we used each of the three simple design similarity measures $P\left(D_{i}, D_{j}\right), Q\left(D_{i}, D_{j}\right)$ and $R\left(D_{i}, D_{j}\right)$ to identify a similar existing product design and evaluated those fixtures (the best available fixtures). Table 2 includes these also. Note that, in some cases, the design similarity measure identified an existing product design whose fixture could not hold the new product design. In these cases, the usefulness is zero.

Then, for each new product design, we compared the usefulness of the best available fixtures to the usefulness of the optimal fixture. We constructed the optimal fixture using existing generative fixture planning approaches (Brost and Goldberg 1996, Zhuang and Goldberg 1996). Table 3 shows the relative values to facilitate comparison. These results show that, compared with the simple design similarity measures, the specialized design similarity measure identifies a product design whose fixture has a higher usefulness metric on average and in nine of the ten cases. Of course, none of the design similarity measures can find the optimal fixture, which is not in the database. This is an inherent limitation of the variant approach.

\subsection{Example}

The following example illustrates the variant fixture planning approach. Consider the new product design shown in figure 1. Figure 2 pictures the design, from the set of existing product designs and fixtures, that is most similar, according to the specialized design similarity measure. For the new product design and the existing fixture triplet, there are multiple feasible configurations and clamp positions.

\begin{tabular}{|c|c|c|c|c|c|}
\hline \multirow[b]{2}{*}{$\begin{array}{l}\text { New } \\
\text { product }\end{array}$} & \multicolumn{4}{|c|}{$\begin{array}{l}\text { Fixture usefulness for the fixture of the most similar } \\
\text { design identified by the design similarity measure }\end{array}$} & \multirow{2}{*}{$\begin{array}{l}\text { Fixture } \\
\text { usefulness } \\
\text { of the } \\
\text { optimal } \\
\text { fixture }\end{array}$} \\
\hline & $\begin{array}{l}P\left(D_{i}, D_{j}\right) \\
\quad(\text { MID })\end{array}$ & $\begin{array}{l}Q\left(D_{i}, D_{j}\right) \\
(\text { TEA })\end{array}$ & $\begin{array}{l}R\left(D_{i}, D_{j}\right) \\
(\mathrm{MVED})\end{array}$ & $S\left(D_{i}, D_{j}\right)$ & \\
\hline 1 & 7.370 & 0 & 11.399 & 14.629 & 69.292 \\
\hline 2 & 0.975 & 0 & 21.617 & 22.344 & 69.717 \\
\hline 3 & 0 & 0 & 0 & 7.832 & 13.229 \\
\hline 4 & 0 & 0 & 0 & 16.148 & 33.773 \\
\hline 5 & 0 & 15.595 & 15.584 & 28.454 & 33.549 \\
\hline 6 & 0 & 0 & 0 & 0.695 & 23.366 \\
\hline 7 & 3.768 & 0 & 0 & 0 & 36.504 \\
\hline 8 & 13.977 & 13.977 & 13.977 & 16.726 & 16.726 \\
\hline 9 & 15.426 & 0 & 21.127 & 21.127 & 28.253 \\
\hline 10 & 0 & 0 & 0 & 7.360 & 8.334 \\
\hline
\end{tabular}

Table 2. Ten new product designs and the best available fixtures. 
Relative fixture usefulness for the fixture of the most similar design identified by the design similarity measure

\begin{tabular}{cllll}
$\begin{array}{c}\text { New } \\
\text { product }\end{array}$ & $\begin{array}{c}P\left(D_{i}, D_{j}\right) \\
(\text { MID })\end{array}$ & $\begin{array}{c}Q\left(D_{i}, D_{j}\right) \\
(\text { TEA })\end{array}$ & $\begin{array}{c}R\left(D_{i}, D_{j}\right) \\
(\text { MVED })\end{array}$ & $S\left(D_{i}, D_{j}\right)$ \\
\hline 1 & 0.1064 & 0 & 0.1645 & 0.2111 \\
2 & 0.3009 & 0 & 0.3101 & 0.3205 \\
3 & 0 & 0 & 0 & 0.5920 \\
4 & 0 & 0 & 0 & 0.4781 \\
5 & 0 & 0.4648 & 0.4645 & 0.8481 \\
6 & 0 & 0 & 0 & 0.0297 \\
7 & 0.1032 & 0 & 0 & 0 \\
8 & 0.8356 & 0.8356 & 0.8356 & 1.0000 \\
9 & 0.5460 & 0 & 0.7478 & 0.7478 \\
10 & 0 & 0 & 0 & 0.8831 \\
Average & 0.1892 & 0.1300 & 0.2523 & 0.5111 \\
\hline
\end{tabular}

Table 3. Relative fixture usefulness of the best available fixtures.

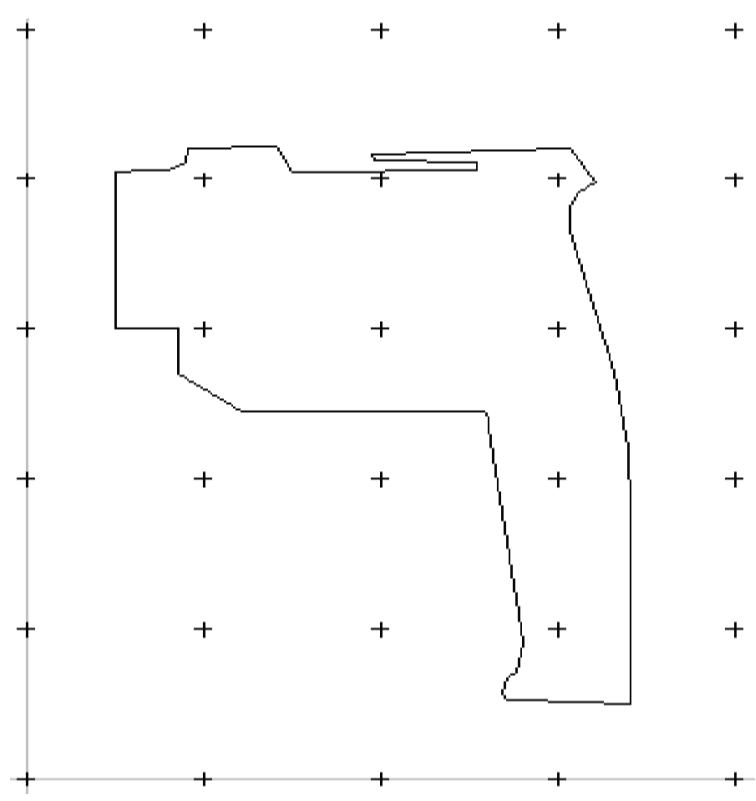

Figure 1. New product design.

Figure 3 pictures the best configuration and clamp position. The corresponding torque resistance metric equals 18.356 .

In this domain, we can construct the optimal fixture using existing generative fixture planning approaches (Brost and Goldberg 1996, Zhuang and Goldberg 1996). Figure 4 shows the optimal fixture. The corresponding torque resistance metric equals 24.872. Thus, the variant fixture planning approach found an available fixture whose relative usefulness equals 0.74 . 


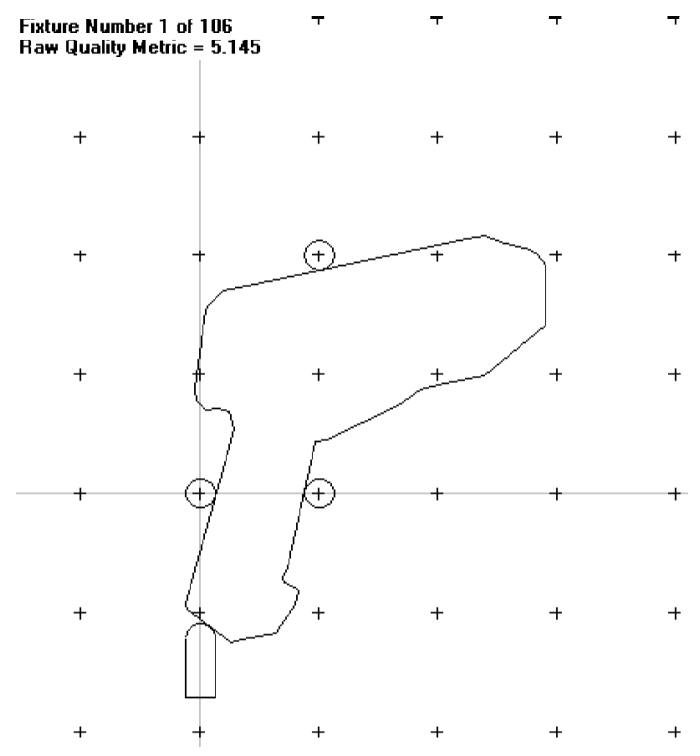

Figure 2. Existing product design and existing fixture.

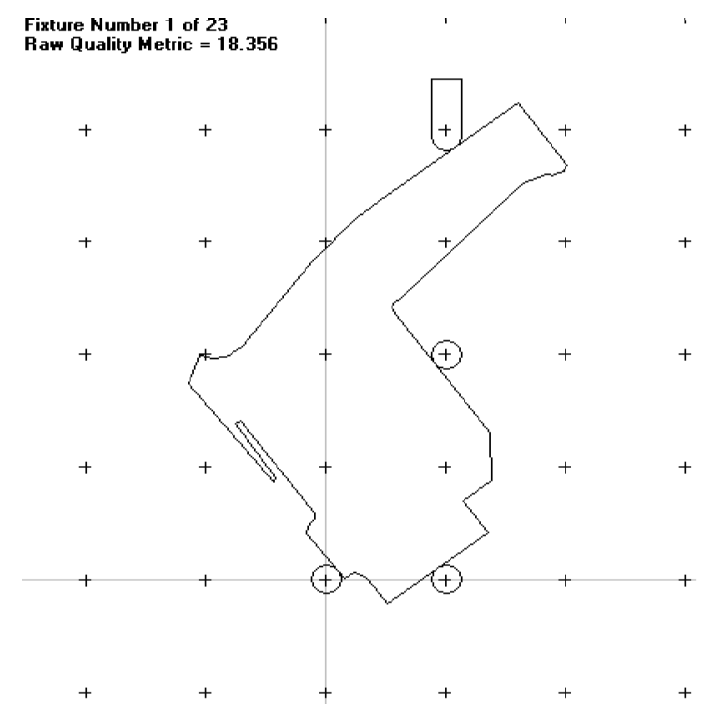

Figure 3. New product design in existing fixture.

\section{Summary and conclusions}

Identifying similar product designs is an important task when making decisions about a new product design. A manufacturing engineer can use a similar product design to generate the process plan, to design the fixture, or to estimate the manufacturing cost of the new product design. Identifying similar product designs effectively and quickly requires good design similarity measures. However, existing design similarity measures can be inconsistent and inappropriate tools, which leads to poor decisions and unnecessary effort to find a better solution. 


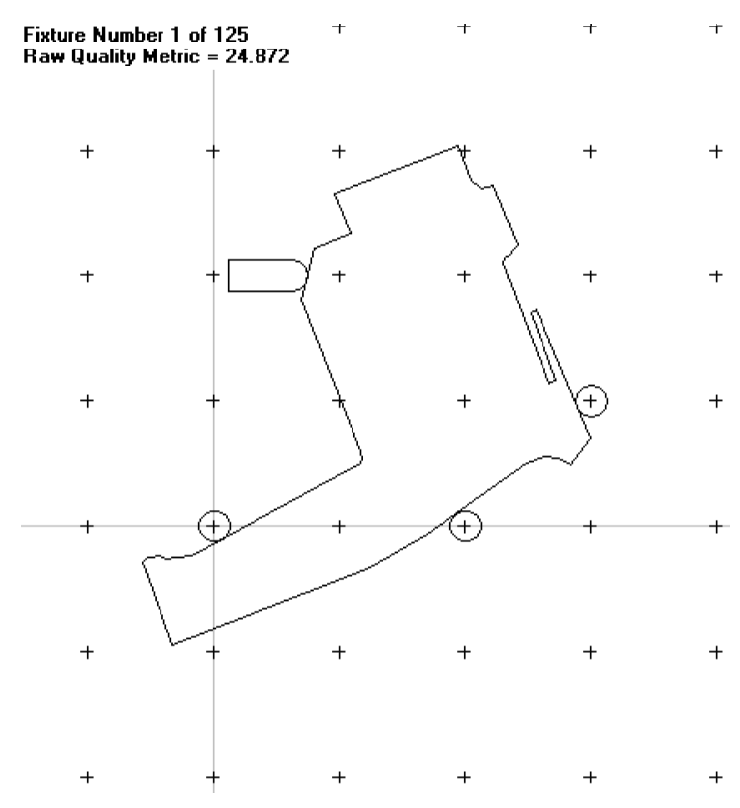

Figure 4. New product design and optimal fixture.

This paper discusses an approach to define a specialized design similarity measure that can help decision-makers find good solutions quickly. This approach uses an artificial neural network. The set of existing product designs and information is used to train the neural network, which learns the specialized design similarity measure. Specialized design similarity measures will be useful in variant and casebased procedures, such as those for process planning, fixture planning, and manufacturability evaluation.

We have applied this approach to a specific variant fixture planning domain. The neural network generates a design similarity measure that finds more appropriate existing designs than design similarity measures based on individual product attributes. The better design similarity measure leads to better fixtures.

Note that this approach requires an expert to define the important product design attributes and the usefulness function. The approach is practical only if the database of available product design attributes is available and the usefulness function for pairs of product designs can be calculated automatically, since training the neural network requires this data. If this data is not available, it may be more practical to use an expert system approach like that described in Section 2.2.

While this approach will be an important part of hybrid process planning, future work in that area needs to consider more complex fixture planning problems.

\section{Acknowledgements}

This paper describes work that was supported by the Office of Naval Research and by the National Science Foundation under grant DMI-97-13718. We would like to thank our colleagues Dana Nau, Ioannis Minis and Alex Elinson for many useful discussions. 


\section{Appendix A: Design similarity measures}

In this fixture planning domain, the product design attributes of interest were the Maximum Inter-vertex Distance (MID), the Maximum Vertex-Edge Distance (MVED), and the Total Enclosed Area (TEA). All of these are functions of the two-dimensional projection of the product design. The MID is the maximum distance between any pair of vertices in the polygon. The TEA is the area enclosed by the polygon. The MVED is the maximum distance between a vertex and a line containing an edge of the polygon. We developed three single-attribute design similarity measures. Each measure was based on one of the following three product design attributes: MID, TEA or MVED.

Let $P\left(D_{0}, D_{j}\right)$ be the MID-based similarity of product design $D_{j}$ to product design $D_{0}$, where $\operatorname{MID}(D)$ represents the MID of product design $D$.

$$
\begin{aligned}
& P\left(D_{0}, D_{j}\right)=\exp \left(-\left|\operatorname{MID}\left(D_{0}\right) / \operatorname{MID}\left(D_{j}\right)-1\right|\right) \text { if } \operatorname{MID}\left(D_{0}\right) / \operatorname{MID}\left(D_{j}\right) \geq 0.9 \\
& P\left(D_{0}, D_{j}\right)=0 \text { otherwise. }
\end{aligned}
$$

Thus, $P\left(D_{0}, D_{j}\right)=1$ if and only if $\operatorname{MID}\left(D_{0}\right)=\operatorname{MID}\left(D_{j}\right)$. Otherwise, $P\left(D_{0}, D_{j}\right)$ decreases (and approaches zero) as $\operatorname{MID}\left(D_{0}\right)$ and $\operatorname{MID}\left(D_{j}\right)$ get farther apart. See figure 5. If $\operatorname{MID}\left(D_{0}\right)$ is significantly smaller than $\operatorname{MID}\left(D_{j}\right)$, then $P\left(D_{0}, D_{j}\right)=0$ because a small part will not fit into a fixture for a large product design.

Similarly, we can define a TEA-based design similarity measure $Q\left(D_{0}, D_{j}\right)$ and a MVED-based design similarity measure $R\left(D_{0}, D_{j}\right)$. TEA $(D)$ represents the TEA of product design $D$. MVED $(D)$ represents the MVED of product design $D$.

$$
\begin{aligned}
Q\left(D_{0}, D_{j}\right) & =\exp \left(-\left|\operatorname{TEA}\left(D_{0}\right) / \operatorname{TEA}\left(D_{j}\right)-1\right|\right) \text { if } \operatorname{TEA}\left(D_{0}\right) / \operatorname{TEA}\left(D_{j}\right) \geq 0.9 \\
Q\left(D_{0}, D_{j}\right) & =0 \text { otherwise. } \\
R\left(D_{0}, D_{j}\right) & =\exp \left(-\left|\operatorname{MVED}\left(D_{0}\right) / \operatorname{MVED}\left(D_{j}\right)-1\right|\right) \text { if } \operatorname{MVED}\left(D_{0}\right) / \operatorname{MVED}\left(D_{j}\right) \\
& \geq 0.9 \\
R\left(D_{0}, D_{j}\right) & =0 \text { otherwise. }
\end{aligned}
$$

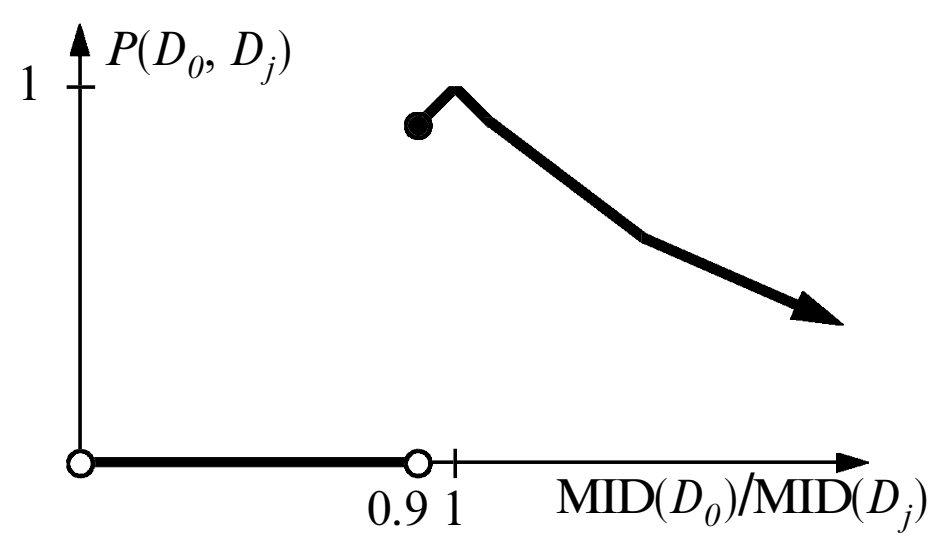

Figure 5. Single-attribute design similarity measure. 


\section{References}

Alting, L. and ZhANG, H., 1989, Computer aided process planning: the state-of-the-art survey. International Journal of Production Research, 27, 553-585.

Balasubramanian, S., Elinson, A., Herrmann, J. W. and Nau, D ., 1998, Fixture-based usefulness measures for hybrid process planning. Proceedings of the 1998 ASME Design for Manufacturing Conference, Atlanta, Georgia, 13-16 September.

Bedworth, D. D., Henderson, M. R., And Wolfe, P. M., 1991, Computer-Integrated Design and Manufacturing (Boston: McGraw-Hill).

BisHoP, C.M., 1995, Neural Networks for Pattern Recognition (New York: Oxford University Press).

Brost, R.C. and GoldBERG, K.Y., 1996, A complete algorithm for designing planar fixtures using modular components. IEEE Transactions on Robotics and Automation, 12 (February), 31-46.

Burbidge, J. L., 1989, Production Flow Analysis for Planning Group Technology (Oxford: Clarendon Press).

CAndadai, A., Herrmann, J. W. and Minis, I., 1995, A group technology-based variant approach for agile manufacturing. Concurrent Product and Process Engineering, International Mechanical Engineering Congress and Exposition, San Francisco, California, 12-17 November; 1996, Applications of group technology in distributed manufacturing, Journal of Intelligent Manufacturing, 7, 271-291.

CHANG, C.-H., 1992, Computer-assisted fixture planning for machining processes. Manufacturing Review, 5, 15-28.

Chang,T.-C. and Wysk, R. A., 1985, An Introduction to Automated Process Planning (Englewood Cliffs, New Jersey: Prentice-Hall).

Chou, Y.-C., SRinivas, R. A. and SARAF, S., 1994, Automatic design of machining fixtures: conceptual design. International Journal of Advanced Manufacturing Technology, 9, 312.

Computer Aided Manufacturing Laboratory, 1979, Part Family Classification and Coding, Monograph Number 3, Brigham Young University, Provo, Utah.

D ARVISHI, A. R . and GILL, K. F., 1990, Expert system rules for fixture design. International Journal of Production Research, 28, 1901-1920.

Elinson, A., NAU, D. S. and REGLI, W. C., 1997, Feature-based similarity assessment of solid models. Proceedings of the ACM Solid Modeling Conference, Atlanta, Georgia, May.

Fuh, J. Y. H., Chang, C.-H . and MelkanofF, M. A., 1993, An integrated fixture planning and analysis system for machining processes. Robotics \& Computer-Integrated Manufacturing, 10, 339-353.

Gupta, S. K., NAU, D.S., Regli, W.C. and Zhang, G., 1994, A methodology for systematic generation and evaluation of alternative operation plans. In Advances in Feature Based Manufacturing, edited by Jami Shah, Martti Mantyla, and Dana Nau (Elsevier/North Holland), pp. 161-184.

Halevi,G. and Weill, R. D.,1995, Principles of Process Planning (New York: Chapman \& Hall).

Hargrove, S. K. and Kusiak, A., 1994, Computer-aided fixture design: a review. International Journal of Production Research, 32, 733-753.

Hassoun, M. H., 1995, Fundamentals of Artificial Neural Networks (Cambridge, MA: The MIT Press).

HERRMANN, J. W . and Singh, G., 1997, Design similarity measures for process planning and design evaluation. Technical Report 97-74, Institute for Systems Research, University of Maryland, College Park.

HofFMAN, E. G., 1984, Fundamentals of Tool Design (Englewood Cliffs, New Jersey: PrenticeHall); 1987, Modular Fixturing (Lake Geneva, Wisconsin: Manufacturing Technology Press).

Hounshell, D. A., 1984, From the American System to Mass Production, 1800-1932 (Baltimore, Maryland: The Johns Hopkins University Press).

HOUTZEEL, A. and SCHILPEROORT, M., 1976, A chain-structured part classification system (MICLASS) and group technology. Proceedings of the 13th Annual Meeting and Technical Conference, Cincinnati, Ohio, March, pp. 383-400. 
Huang, C.-L., Huang, Y .-H ., Chang, T.-Y., Chang, S.-H., Chung, C.-H ., HuAng, D .-T. and LI, R.-K., 1999, The construction of production performance prediction system for semiconductor manufacturing with artificial neural networks. International Journal of Production Research, 37, 1387-1402.

IYER, S. and NAGI, R., 1994, Identification and ranking of similar parts in agile manufacturing. In Concurrent Product Design, edited by R. Gadh (New York: ASME) pp. 87-96; 1997, Automated retrieval and ranking of similar parts in agile manufacturing. IIE Transactions, 29, 859-876.

KING, J. R., 1980, Machine-component grouping in production flow analysis: an approach using rank ordering clustering algorithm. International Journal of Production Research, 18,213-237.

Kumar, S. A., NeE, A. Y. C. and Prombanpong, S., 1992, Expert fixture-design system for an automated manufacturing environment. Computer-aided Design, 24, 317-326.

KUSIAK, A., 1987, The generalized group technology concept. International Journal of Production Research, 25, 561-569.

LEUnG, H. C., Hines, E. L. and RAJA, V. H., 1994, The application of artificial neural networks in computer aided process planning. Intelligent Engineering Systems through Artificial Neural Networks, 4, 1091-1096.

LIPPMANN, R. P., 1987, An introduction to computing with neural nets. IEEE ASSP Magazine, pp. 4-22.

Mitrofanov, S. P., 1996, The Scientific Principles of Group Technology, National Lending Library Translation.

Muller, B. and ReinhaRdT, J., 1990, Neural Networks: An Introduction (Berlin: SpringerVerlag).

OFFODILE, O.F., Application of similarity coefficient method to parts coding and classification analysis in group technology. Journal of Manufacturing Systems, 10, 442-448.

OpITZ, H., 1970, A Classification to Describe Workpieces (Oxford: Pergamon Press).

SNEAD, C. S., 1989, Foundation for Competitive Manufacturing, Competitive Manufacturing Series (Van Nostrand Reinhold).

Sun, T.-L., SU, C.-J., MAYER, R. J. and WYSK, R. A., 1995, Shape similarity assessment of mechanical parts based on solid models. Proceedings of the 1995 Design Engineering Technical Conferences, edited by O. M. Jadaan et al., Volume 83, Number 2, pp. 953962 (New York: ASME).

Trappey, J. C. and LiU, C. R., 1990, A literature survey of fixture-design automation. International Journal of Advanced Manufacturing Technology, 9, 240-255.

WU, M. C. and JEN, S. R., 1996, A neural network approach to the classification of 3D prismatic parts. International Journal of Advanced Manufacturing Technology, 11, 325-335.

Yue, Y. and Murray, J., 1994, Workpiece selection and clamping of complex 2.5D components. in Advances in Feature Based Manufacturing, edited by J. Shah, M. Mäntylä, and D. Nau (Amsterdam: Elsevier), pp. 185-214.

ZhUANG, Y. and GoldBERG, K. Y., 1996, On the existence of solutions in modular fixturing. International Journal of Production Research, 15, 646-656. 